\title{
A New Class of Strongly Summable and Statistical Convergence Sequences of Fuzzy Numbers
}

\author{
Hemen Dutta* \\ Department of Mathematics, Gauhati University, Guwahati-781014, Assam, India
}

Received: 22 Jan. 2013, Revised: 24 May. 2013, Accepted: 27 May. 2013

Published online: 1 Nov. 2013

\begin{abstract}
The paper introduces a new concept of strong summability and statistical convergence of sequence of fuzzy numbers. Further, these two concepts are studied and established relations between them.
\end{abstract}

Keywords: Sequence of fuzzy numbers, difference sequence space, statistical convergence, summability.

\section{Introduction}

The analysis of sequence spaces deals with questions such as the distance between sequences, Cauchy sequences and limits of a sequence of 'points' in the class of all sequences. Studies on sequence space were further extended through summability theory. In its broadest meaning, summability theory, or in short summability, is the theory of the assignment of limits, which is essential and useful in analysis, function theory, topology and applied mathematics.

The idea of the statistical convergence of sequence was introduced by Fast [9] and Schoenberg [18] independently in order to extend the notion of convergence of sequences. It is also found in Zygmund [24]. Later on it was linked with summability by Fridy and Orhan [10], Maddox [13], Rath and Tripathy [17] and many others. Nuray and Savaş [16] extended the idea to sequences of fuzzy numbers and discussed the concept of statistically Cauchy sequences of fuzzy numbers. On strongly $\lambda$-summability and $\lambda$-statistical convergence can be found in [20]. Çakan and Şengönül [6] studied the space of convergent and bounded sequence of fuzzy numbers and showed that they are Banach spaces under a suitable norm. Altmok, Altin and Et [5] introduced the notions of lacunary almost statistical convergence and lacunary strongly almost convergent sequences. Few recent works in this direction on fuzzy mathematics or which can be extended through fuzzy mathematics can be found in ( [1-4], [14]).

Let $C\left(R^{n}\right)=\left\{A \subset R^{n}: A\right.$ compact and convex $\}$. The space $C\left(R^{n}\right)$ has a liner structure induced by the operations $A+B$ $=\{a+b: a \in A, b \in B\}$ and $\lambda A=\{\lambda a: a \in A\}$ for $A$, $B \in C\left(R^{n}\right)$ and $\lambda \in R$. The Hausdroff distance between $A$ and $B$ of $C\left(R^{n}\right)$ is defined as:

$$
\delta_{\infty}(A, B)=\max \left\{\sup _{a \in A} \inf _{b \in B}\|a-b\|, \sup _{b \in B} \inf _{a \in A}\|a-b\|\right\} .
$$

Let $L\left(R^{n}\right)$ denote the set of all fuzzy numbers. The linear structure of $L\left(R^{n}\right)$ induces addition $X+Y$ and scalar multiplication $\lambda X, \lambda \in R$, in terms of $\alpha$-level sets, by $[X+Y]^{\alpha}=[X]^{\alpha}+[Y]^{\alpha}$ and $[\lambda X]^{\alpha}=\lambda[X]^{\alpha}$ for each $0 \leq \alpha \leq$ 1 , where the $\alpha$-level set $[X]^{\alpha}=\left\{x \in R^{n}: X(x) \geq \alpha\right\}$ is a nonempty compact and convex subset of $R^{n}$ and $X$ is a fuzzy number i.e., a function from $R^{n}$ to $[0,1]$ which is normal, fuzzy convex, upper semi-continuous and the closure $X^{0}=\left\{x \in R^{n}: X(x)>0\right\}$ is compact.

Define for each $1 \leq q<\infty$

$$
d_{q}(X, Y)=\left(\int_{0}^{1} \delta_{\infty}\left(X^{\alpha}, Y^{\alpha}\right)^{q} d_{\infty}\right)^{1 / q}
$$

and

$$
d_{\infty}=\sup _{0 \leq \alpha \leq 1} \delta_{\infty}\left(X^{\alpha}, Y^{\alpha}\right) .
$$

Clearly

$d_{\infty}(X, Y)=\lim _{q \rightarrow \infty} d_{q}(X, Y)$ with $d_{q} \leq d_{r}$ if $q \leq r$. Moreover $d_{q}$ is a complete, separable and locally compact metric

\footnotetext{
*Corresponding author e-mail: hemen_dutta08@ rediffmail.com
} 
space (see [7]).

Throughout the paper, $d$ will denote $d_{q}$ with $1 \leq q<\infty$.

We now state the following definitions which can be found in ( [12], [16], [19]).

A sequence $X=\left(X_{k}\right)$ of fuzzy numbers is a function $X$ from the set $N$ of all positive integers into $L(R)$. The fuzzy number $X_{k}$ denotes the value of the function at $k \in N$ and is called the $k$-th term or general term of the sequence.

A sequence $X=\left(X_{k}\right)$ of fuzzy numbers is said to be convergent to the fuzzy number $X_{0}$, written as $\lim _{k} X_{k}=$ $X_{0}$, if for every $\varepsilon>0$ there exists $n_{0} \in N$ such that

$d\left(X_{k}, X_{0}\right)<\varepsilon$ for $k>n_{0}$

Again $X=\left(X_{k}\right)$ is said to be a Cauchy sequence if for every $\varepsilon>0$ there exists $n_{0} \in N$ such that

$d\left(X_{k}, X_{l}\right)<\varepsilon$ for $k, l>n_{0}$

A sequence $X=\left(X_{k}\right)$ of fuzzy numbers is said to be bounded if the set $\left\{X_{k}: k \in N\right\}$ of fuzzy numbers is bounded.

The natural density of a set $K$ of positive integers is denoted by $\delta(K)$ and defined by

$$
\delta(K)=\lim _{n} \frac{1}{n} \operatorname{card}\{k \leq n: k \in K\}
$$

If $X=\left(X_{k}\right)$ is a sequence that satisfies a property say, $P$ for all $k$ except on a set of natural density zero, we say that $X_{k}$ satisfies $P$ for almost all $k$ and we write it by 'a.a.k'.

A sequence $X=\left(X_{k}\right)$ of fuzzy numbers is said to be statistically convergent to a fuzzy number $X_{0}$ if for every $\varepsilon>0, \quad \lim _{n} \frac{1}{n} \operatorname{card}\left\{k \leq n: d\left(X_{k}, X_{0}\right) \geq \varepsilon\right\}=0$, i.e., $d\left(X_{k}, X_{0}\right)<\varepsilon$ a.a. $k$. We write st-lim $X_{k}=X_{0}$.

The notion of difference sequence space was introduced by Kizmaz [11], who studied the difference complex or real sequence spaces $\ell_{\infty}(\Delta), c(\Delta)$ and $c_{0}(\Delta)$. The notion was further generalized by Et and Colak [8] by introducing the spaces $\ell_{\infty}\left(\Delta^{s}\right), c\left(\Delta^{s}\right)$ and $c_{0}\left(\Delta^{s}\right)$. Another type of generalization of the difference sequence spaces is due to Tripathy and Esi [22], who studied the spaces $\ell_{\infty}\left(\Delta_{r}\right), \quad c\left(\Delta_{r}\right)$ and $c_{0}\left(\Delta_{r}\right)$. Tripathy, Esi and Tripathy [23] generalized the above notions and unified these as follows:

Let $r, s$ be non- negative integers, then for $Z$ a given sequence space we have

$$
Z\left(\Delta_{r}^{s}\right)=\left\{x=\left(x_{k}\right) \in w:\left(\Delta_{r}^{s} x_{k}\right) \in Z\right\},
$$

where $\Delta_{r}^{s} x=\left(\Delta_{r}^{s} x_{k}\right)=\left(\Delta_{r}^{s-1} x_{k}-\Delta_{r}^{s-1} x_{k+r}\right)$ and $\Delta_{r}^{0} x_{k}=x_{k}$ for all $k \in N$, which is equivalent to the following binomial representation

$$
\Delta_{r}^{s} x_{k}=\sum_{v=0}^{s}(-1)^{v}\left(\begin{array}{l}
s \\
v
\end{array}\right) x_{k+r v} .
$$

In this article, we define the difference operator $\Delta_{(v, r)}^{s}$ for sequences of fuzzy numbers and we will describe it in the next section. This new difference operator generalizes all previous difference operators.

2 Strongly $\left(\Delta_{(v, r)}^{s}\right)^{\lambda p}$ - Summable and $\left(\Delta_{(v, r)}^{s}\right)^{\lambda p}$ - Statistical Convergence

Let $r$ and $s$ be two non-negative integers and $v=\left(v_{k}\right)$ be a sequence of non-zero reals. Let $\lambda=\left(\lambda_{k}\right)$ be a non-decreasing sequence of positive numbers tending to $\infty$ and $\lambda_{n+1}=\lambda_{n}+1, \lambda_{1}=1$. Then for $0<p<\infty$, the sequence $X=\left(X_{k}\right)$ of fuzzy numbers is said to be strongly $\left(\Delta_{(v, r)}^{s}\right)^{\lambda p}$ - summable to a fuzzy number $X_{0}$ if

$\lim _{n \rightarrow \infty} \frac{1}{\lambda_{n}} \sum_{k \in I_{n}} d\left(\Delta_{(v, r)}^{s} X_{k}, X_{0}\right)^{p}=0, \quad$ where $\quad I_{n}=$ $\left[n-\lambda_{n}+1, n\right]$ and

$\left(\Delta_{(v, r)}^{s} X_{k}\right)=\left(\Delta_{(v, r)}^{s-1} X_{k}-\Delta_{(v, r)}^{s-1} X_{k-r}\right)$ and $\Delta_{(v, r)}^{0} X_{k}=v_{k} X_{k}$ for all $k \in N$, which is equivalent to the following binomial representation:

$$
\Delta_{(v, r)}^{s} X_{k}=\sum_{i=0}^{s}(-1)^{i}\left(\begin{array}{c}
s \\
i
\end{array}\right) v_{k-r i} X_{k-r i} .
$$

In this expansion, we take $v_{k}=0$ and $X_{k}=\overline{0}$ for non-positive values of $k$.

Taking $s=0$ and $v_{k}=1$, for all $k \in N$, then strongly $\left(\Delta_{(v, r)}^{s}\right)^{\lambda p}$-summability reduces to strongly $\lambda p$-summability. It is clear that strongly $\lambda p$-summability implies strongly $\left(\Delta_{(v, r)}^{s}\right)^{\lambda p}$-summability.

In particular, if we take $\lambda_{n}=n$, for all $n \in N$ then we say $X$ $=\left(X_{k}\right)$ is strongly $\left(\Delta_{(v, r)}^{s}\right)^{p}$ - Cesàro summable to $X_{0}$.

Again by taking $s=0$ and $v_{k}=1$, for all $k \in N$ strongly $\left(\Delta_{(v, r)}^{s}\right)^{p}$ - Cesàro summability reduces to strongly p-Cesàro summability.

A sequence $X=\left(X_{k}\right)$ of fuzzy numbers is said to be $\left(\Delta_{(v, r)}^{s}\right)^{\lambda p}$ - statistically convergent to a fuzzy number $X_{0}$ 
if for every $\varepsilon>0$

$$
\lim _{n} \frac{1}{\lambda_{n}} \operatorname{card}\left\{k \in I_{n}: d\left(\Delta_{(v, r)}^{s} X_{k}, X_{0}\right)^{p} \geq \varepsilon\right\}=0
$$

In particular, if we take $\lambda_{n}=n$, for all $n \in N$, then we say that it is $\left(\Delta_{(v, r)}^{s}\right)^{p}$ - statistically convergent to $X_{0}$.

Again if we take $\lambda_{n}=n$, for all $n \in N, s=0, p=1$ and $v_{k}=$ 1 , for all $k \in N$, then $\left(\Delta_{(v, r)}^{s}\right)^{\lambda p}$ - statistically convergence reduces to statistically convergence.

Now we present some relationship between strongly $\left(\Delta_{(v, r)}^{s}\right)^{\lambda p}$ - summability and $\left(\Delta_{(v, r)}^{s}\right)^{\lambda p}$ - statistically convergent.

Theorem 2.1. If a sequence $\mathrm{X}=\left(X_{k}\right)$ is strongly $\left(\Delta_{(v, r)}^{s}\right)^{\lambda p}$ - summable then it is $\left(\Delta_{(v, r)}^{s}\right)^{\lambda p}$ - statistically convergent.

Proof. Suppose $X=\left(X_{k}\right)$ is strongly $\left(\Delta_{(v, r)}^{s}\right)^{\lambda p}$ - summable to $X_{0}$. Then

$$
\lim _{n \rightarrow \infty} \frac{1}{\lambda_{n}} \sum_{k \in I_{n}} d\left(\Delta_{(v, r)}^{s} X_{k}, X_{0}\right)^{p}=0
$$

It is easy to see that

$\sum_{k \in I_{n}} d\left(\Delta_{(v, r)}^{s} X_{k}, X_{0}\right)^{p} \geq \varepsilon \operatorname{card}\left\{k \in I_{n}: d\left(\Delta_{(v, r)}^{s} X_{k}, X_{0}\right)^{p} \geq \varepsilon\right\}$

This gives the result.

Theorem 2.2.If a sequence $\mathrm{X}=\left(X_{k}\right)$ is $\Delta_{(v, r)}^{s}$ - bounded and $\left(\Delta_{(v, r)}^{s}\right)^{\lambda}$ - statistically convergent then it is strongly $\left(\Delta_{(v, r)}^{s}\right)^{\lambda p}$-summable.

Proof. Suppose $X=\left(X_{k}\right)$ is $\Delta_{(v, r)}^{s}$-bounded and $\left(\Delta_{(v, r)}^{s}\right)^{\lambda}$ statistically convergent to $X_{0}$. Since $X=\left(X_{k}\right)$ is $\Delta_{(v, r)}^{s}$-bounded, we can find a fuzzy number $M$ such that

$$
d\left(\Delta_{(v, r)}^{s} X_{k}, X_{0}\right)^{p} \leq M
$$

for all $k \in N$

Again since $X=\left(X_{k}\right)$ is $\left(\Delta_{(v, r)}^{s}\right)^{\lambda}$ - statistically convergent to $X_{0}$, we have

$$
\lim _{n} \frac{1}{\lambda_{n}} \operatorname{card}\left\{k \in I_{n}: d\left(\Delta_{(v, r)}^{s} X_{k}, X_{0}\right)^{p} \geq \varepsilon\right\}=0
$$

for every $\varepsilon>0$.
Now we can easily verify the followings:

$$
\begin{aligned}
& \frac{1}{\lambda_{n}} \sum_{k \in I_{n}} d\left(\Delta_{(v, r)}^{s} X_{k}, X_{0}\right)^{p} \\
& \frac{1}{\lambda_{n}} \sum_{k \in I_{n}, d\left(\Delta_{(v, r)}^{s} X_{k}, X_{0}\right)^{p} \geq \varepsilon} d\left(\Delta_{(v, r)}^{s} X_{k}, X_{0}\right)^{p} \\
& \quad+\frac{1}{\lambda_{n}} \sum_{k \in I_{n}, d\left(\Delta_{(v, r)^{s}}^{s} X_{k}, X_{0}\right)^{p}<\varepsilon} d\left(\Delta_{(v, r)}^{s} X_{k}, X_{0}\right)^{p} \\
& \quad \leq \frac{M}{\lambda_{n}} \operatorname{card}\left\{k \in I_{n}: d\left(\Delta_{(v, r)}^{s} X_{k}, X_{0}\right)^{p} \geq \varepsilon\right\}+\varepsilon
\end{aligned}
$$

This completes the proof.

Corollary 2.1. If a sequence $\mathrm{X}=\left(X_{k}\right)$ is $\Delta_{(v, r)}^{s}$-bounded and $\left(\Delta_{(v, r)}^{s}\right)^{\lambda}$ - statistically convergent then it is strongly $\left(\Delta_{(v, r)}^{s}\right)^{p}$-Cesàro summable.

Proof. The proof is a consequence of the above theorem and the following inequality:

$$
\begin{aligned}
& \frac{1}{n} \sum_{k=1}^{n} d\left(\Delta_{(v, r)}^{s} X_{k}, X_{0}\right)^{p} \\
& \frac{1}{n} \sum_{k=1}^{n-\lambda_{n}} d\left(\Delta_{(v, r)}^{s} X_{k}, X_{0}\right)^{p}+\frac{1}{n} \sum_{k \in I_{n}} d\left(\Delta_{(v, r)}^{s} X_{k}, X_{0}\right)^{p} \\
& \leq \frac{1}{\lambda_{n}} \sum_{k=1}^{n-\lambda_{n}} d\left(\Delta_{(v, r)}^{s} X_{k}, X_{0}\right)^{p}+\frac{1}{\lambda_{n}} \sum_{k \in I_{n}} d\left(\Delta_{(v, r)}^{s} X_{k}, X_{0}\right)^{p} \\
& \quad \leq \frac{2}{\lambda_{n}} \sum_{k \in I_{n}} d\left(\Delta_{(v, r)}^{s} X_{k}, X_{0}\right)^{p}
\end{aligned}
$$

Theorem 2.3. If a sequence $\mathrm{X}=\left(X_{k}\right)$ is $\Delta_{(v, r)}^{s}$-statistically convergent and $\liminf _{n}\left(\frac{\lambda_{n}}{n}\right)>0$ then it is $\left(\Delta_{(v, r)}^{s}\right)^{\lambda p}$-statistically convergent.

Proof. Assume the given conditions. For a given $\varepsilon>0$, we have

$$
\left\{k \in I_{n}: d\left(\Delta_{(v, r)}^{s} X_{k}, X_{0}\right)^{p} \geq \varepsilon\right\} \subset\left\{k \leq n: d\left(\Delta_{(v, r)}^{s} X_{k}, X_{0}\right)^{p} \geq \varepsilon\right\}
$$

Hence the proof follows from the following inequality:

$$
\begin{aligned}
& \frac{1}{n} \operatorname{card}\left\{k \leq n: d\left(\Delta_{(v, r)}^{s} X_{k}, X_{0}\right)^{p} \geq \varepsilon\right\} \\
& \frac{1}{n} \operatorname{card}\left\{k \in I_{n}: d\left(\Delta_{(v, r)}^{s} X_{k}, X_{0}\right)^{p} \geq \varepsilon\right\} \\
& \quad=\frac{\lambda_{n}}{n} \frac{1}{\lambda_{n}} \operatorname{card}\left\{k \in I_{n}: d\left(\Delta_{(v, r)}^{s} X_{k}, X_{0}\right)^{p} \geq \varepsilon\right\}
\end{aligned}
$$

\section{Acknowledgement}

The author is grateful to the anonymous referees for a careful reading of the paper and encouraging comments on it. 


\section{References}

[1] A. Alotaibi and M. Mursaleen, A-Statistical Summability of Fourier Series and Walsh-Fourier Series, Appl. Math. Inf. Sci. 6, 535-538 (2012).

[2] H. Dutta, An application of lacunary summability method to n-norm, Int. J. Appl. Math. Stat., 15, 89-97 (2009).

[3] H. Dutta, On some complete metric spaces of strongly summable sequences of fuzzy numbers, Rend. Semin. Mat. Univ. Politec. Torino, 68, 29-36 (2010).

[4] H. Dutta and T. Bilgin, Strongly $\left(V^{\lambda}, A, \Delta_{(v m)}^{n}, p\right)-$ summable sequence spaces defined by an Orlicz function, Appl. Math. Letters 24, 1057-1062 (2011).

[5] H. Altmok, Y. Altin and M. Et, Lacunary almost statistical convergence of fuzzy numbers, Thai J. Math., 2, 265-274 (2004).

[6] C. Çakan and M. Şengönül, The Ordinary and almost convergence of sequences of fuzzy numbers, Thai J. Math., 2, 1-7 (2004).

[7] P. Diamond and P. Kloeden, Metric spaces of fuzzy sets, Fuzzy Sets and Systems, 35, 241-249 (1990).

[8] M. Et and R. Colak, On generalized difference sequence spaces, Soochow J. Math., 21, 377-386 (1995).

[9] H. Fast, Sur la convergence statistique, Colloq. Math., 241244 (1995).

[10] J. A. Fridy and C. Orhan, Statistical limit superior and limit inferior, Proc. Amer. Math. Soc., 125, 3625-3631 (1997).

[11] H. Kizmaz, On certain sequence spaces, Canad. Math. Bull., 24, 168-176 (1981).

[12] M. Matloka, Sequences of fuzzy numbers, BUSEFAL, 28, 28-37 (1986).

[13] I. J. Maddox, A Tauberian condition for statistical convergence, Math. Proc. Camb. Phil. Soc., 106, 277-280 (1989).

[14] S. Debnath, J. Debnath, Some generalized statistical convergent sequence spaces of fuzzy numbers via ideals, Mathematical Sciences Letters, 2, 151-154 (2013).

[15] S. Nanda, On sequence of fuzzy numbers, Fuzzy Sets and Systems, 33, 28-37 (1989).

[16] F. Nuray and E. Savaş, Statistical convergence of sequences of fuzzy numbers, Math. Slovaca, 45, 269-273 (1995).

[17] D. Rath and B. C. Tripathy, Matrix maps on sequence spaces associated with sets of integers, Indian J. Pure Appl. Math., 27, 197-206 (1996).

[18] I. J. Schoenberg, The integrability of certain functions and related summability methods, Amer. Math. Monthly, 66, 3621-4375 (1959).

[19] E. Savaş, A note on sequence of fuzzy numbers, Inform. Sciences, 124, 297-300 (2000).

[20] E. Savaş, On strongly $\lambda$-summable sequences of fuzzy numbers, Inform. Sciences, 125, 181-186 (2000).

[21] L. A. Zadeh, Fuzzy sets, Inform. and Control, 8, 338-353 (1965).

[22] B. C. Tripathy and A. Esi, A new type of difference sequence spaces, Int. J. Sci. Technol., 1, 11-14 (2006).

[23] B. C. Tripathy, A. Esi and B. K. Tripathy, On a new type of generalized difference Cesro Sequence spaces, Soochow J. Math., 31, 333-340 (2005).

[24] A. Zygmund, Trigonometric series, Cambridge, 2, 1993.

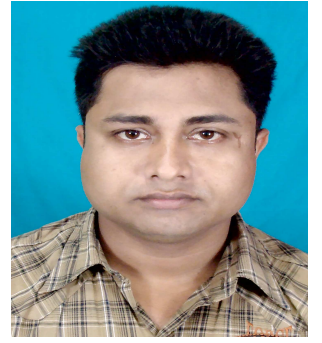

Hemen Dutta is Assistant Professor of Mathematics at Gauhati University, Guwahati, India. His research interests are in the areas of Functional Analysis and Fuzzy Mathematics. He has published research articles in reputed international journals of mathematical sciences. He is referee and editor of mathematical journals. 